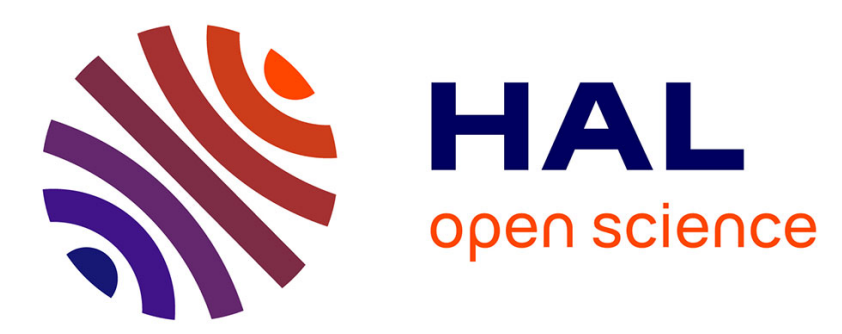

\title{
Experimental and numerical study of the Coanda effect used to reduce self-sustained tones
}

\author{
Cyrille Allery, Sébastien Guérin, Aziz Hamdouni, Anas Sakout
}

\section{To cite this version:}

Cyrille Allery, Sébastien Guérin, Aziz Hamdouni, Anas Sakout. Experimental and numerical study of the Coanda effect used to reduce self-sustained tones. ASME 2002 Joint U.S.-European Fluids Engineering Division Conference, Jul 2002, Québec, Canada. pp.1191-1196, 10.1115/FEDSM200231355 . hal-00312344

\author{
HAL Id: hal-00312344 \\ https://hal.science/hal-00312344
}

Submitted on 18 Dec 2018

HAL is a multi-disciplinary open access archive for the deposit and dissemination of scientific research documents, whether they are published or not. The documents may come from teaching and research institutions in France or abroad, or from public or private research centers.
L'archive ouverte pluridisciplinaire HAL, est destinée au dépôt et à la diffusion de documents scientifiques de niveau recherche, publiés ou non, émanant des établissements d'enseignement et de recherche français ou étrangers, des laboratoires publics ou privés. 


\section{EXPERIMENTAL AND NUMERICAL STUDY OF THE COANDA EFFECT USED TO REDUCE SELF-SUSTAINED TONES}

\author{
C. Allery \\ L3MA \\ Université de Poitiers et E.N.S.M.A \\ B.P. 30179, 86962 Futuroscope Cedex France \\ Email: allery@|3ma.univ-poitiers.fr
}

\author{
S. Guérin \\ LEPTAB \\ Université de La Rochelle \\ 17042 La Rochelle Cedex France \\ Email: sgueri01@univ-Ir.fr
}

\author{
A. Sakout \\ LEPTAB \\ Université de La Rochelle \\ 17042 La Rochelle Cedex France \\ Email: asakout@univ-Ir.fr
}

\section{ABSTRACT}

We present in this paper an experimental and numerical study about the Coanda effect which causes the sudden reattachment of a jet to an inclined plane. This phenomenon induces a large hysteresis loop, which can be used to reduce the noise produced by an airflow crossing two diaphragms in tandem inside a duct. The angle of the inclined wall with horizontal plane and the flow velocity are the two main parameters studied here.

With the aim of doing optimal control, we propose to construct for this flow configuration a low-dimensional dynamical system with a basis issued from a Proper Orthogonal Decomposition.

\section{NOMENCLATURE}

$a(t)$ temporal function of the POD decomposition

$h_{N}$ pipe height

$L$ length of the inclined wall

$L^{2}(\Omega) \quad$ space of functions of finite energy in $\Omega$

$L_{N}$ pipe length

$l_{N}$ pipe width

$n_{o} \quad$ outward normal $\bar{p}, p^{\prime} \quad$ average and fluctuating pressure

$R$ fluctuating spatial correlation tensor

$R e$ Reynolds number

$R e_{c a}, R e_{c d}$ attachment and detachment Reynolds number

$U_{0}$ inlet velocity

$U_{1}, U_{2}$ attachment and detachment velocity

$u(x, t) \quad$ velocity at time $t$ and at $x$ coordinate

$\bar{u}, u^{\prime} \quad$ average and fluctuating velocity

$V$ vertical velocity

$\alpha$ angle of inclination with horizontal plane

$\alpha_{c a}, \alpha_{c d} \quad$ attachment and detachment angle

$\lambda$ eigenvalue of the Fredholm integral equation

$\Omega$ spatial domain

$\phi(x)$ spatial function of the POD decomposition

$\checkmark$ air kinematic viscosity

$(.,$.$) \quad inner product in L^{2}(\Omega)$

\section{INTRODUCTION}

An air flow crossing two diaphragms in tandem inside a pipe may generate intense self-sustained tones when diaphragms are close each other. The sound production results from a feedback 
loop between a vortex shedding and the pipe acoustical resonances. The unstable shear layer created by the upstream diaphragm gives rise indeed to vortices which are convected downstream by the mean flow. These vortices transfer a part of their kinetic energy towards the acoustical field in the vicinity of the downstream diaphragm where they are deviated. The induced acoustical resonant field controls in return the shear layer oscillations which periodically shed new vortices.

In order to break the feedback loop and then to reduce the sound produced, Guérin (2001) created four thin radial diverging slots on the upstream diaphragm at the boundary of the central axisymmetric jet. The auxiliary jets issued from these slots strike directly the vortex rings. They aim to disorganise the coherent vortices before they arrive at the downstream diaphragm where the energetic transfer occurs. He showed that a noise reduction attaining $30 \mathrm{~dB}$ may occur provided to reach the stable path of a bifurcation when velocity varies. Starting from zero, when velocity increases, self-sustained tones are intense as if the upstream diaphragm was unmodified. But, abruptly, at a critical velocity $U_{1}$, the sound pressure level drops. Thereafter, when velocity increases or decreases, the noise remains less intense. It is necessary indeed to decrease the velocity down to a low value $U_{2}\left(U_{2}<<U_{1}\right)$ to have again self-sustained tones when velocity is increased. Measurements of the velocity in the slot wakes show that the phenomenon responsible for the flow reorganisation is a Coanda effect happening on the auxiliary jets (Guérin et al, 2001). When auxiliary jets are axial to the slots, they are weakly efficient to perturb the energetic transfer. But when they are attached by Coanda effect to the slot walls, the self-sustained tones are reduced because auxiliary jets create a swirling flow around the central axisymmetric jet which is efficient to disrupt the energetic transfer. The reattachment of the auxiliary jets to the diverging slot with a hysteresis cycle can be modelled by a jet in interaction with an inclined wall jet. Therefore, we studied this configuration.

The physical explanation of the reattachment of a jet to a wall by Coanda effect is the following. Consider the jet as being fully detached from the plate. Fluid is entrained from the surroundings on its both sides. In the confined region between the jet and the wall, fluid accelerates near the wall and the pressure is reduced there. This pressure drop induces the jet deflection towards the wall and its reattachment if the wall is long enough. Newman (1961) and Lai and Lu (1996) studied experimentally the variation of the reattachment length against the inclination angles $\alpha$ at a constant velocity. They showed for example that this length increases with $\alpha$. At constant velocity and wall length, Newman (1961) found that the jet is always attached to the wall at small angles and never attached at large angles. For intermediate $\alpha$ values, flow is either detached from the wall or reattached to it, depending if the angle is increased or decreased. This result shows a hysteresis cycle. At a constant velocity, Katz (1973) varied the angle $\alpha$ and explored the critical angles at which the

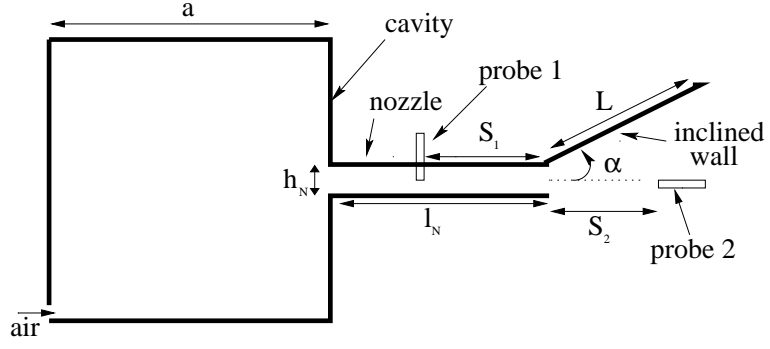

Figure 1. Experimental apparatus

jet becomes attached. At our knowledge, no experiment has been made when the Reynolds number is varied.

In order to control the method of self-sustained tone reduction described above, we study in this paper the model problem of a jet in interaction with an inclined plane. The first part deals with the experimental results about the influence of the Reynolds number and the angle of inclination on the jet reattachment to a plane. The second part is a numerical study. Firstly, results given by LES simulations are compared with the experimental data. Then, we discuss the possibility of constructing a low-dimensional dynamical system, characterising the flow through a divergent by using the Proper Orthogonal Decomposition method (POD). This technique, introduced in fluid mechanics by Lumley (1967), consists in constructing a "physical basis" onto which the Navier-Stokes equations are projected by the Galerkin method. With this basis, it turns out that a few degrees of freedom are necessary to well describe the flow. In this case, the velocity and pressure fields obtained by LES would be used to apply the POD. Finally, this method is validated for a lid driven cavity.

\section{EXPERIMENTAL STUDY Apparatus}

We studied experimentally an incompressible turbulent airflow in interaction with an inclined flat plat. The airflow is supplied by a blower driven by an electrical motor. It goes through a plenum $(a=1000 \mathrm{~mm})$ equipped with honeycomb and screens, then a square duct with length $L_{N}=1000 \mathrm{~mm}$, width $l_{N}=171 \mathrm{~mm}$ and height $h_{N}=10 \mathrm{~mm}$, and finally exits at the atmosphere. So the nozzle aspect ratio $l_{N} / h_{N}$ is 17.1 . The wall length is $L=500 \mathrm{~mm}$ giving $L / h_{N}=50$ and the angle $\alpha$ with horizontal plane varies between $20^{\circ}$ and $50^{\circ}$.

Bradshaw and Won (1972) found that the effect of the nozzle ratio on the reattachment length was negligible for aspect ratio larger than 10. In our experiences $l_{N} / h_{N}>10$ and no side plates were used to enhance the two-dimensionality of the flow.

Measurements use two DANTEC hot wires: the first one, lo- 


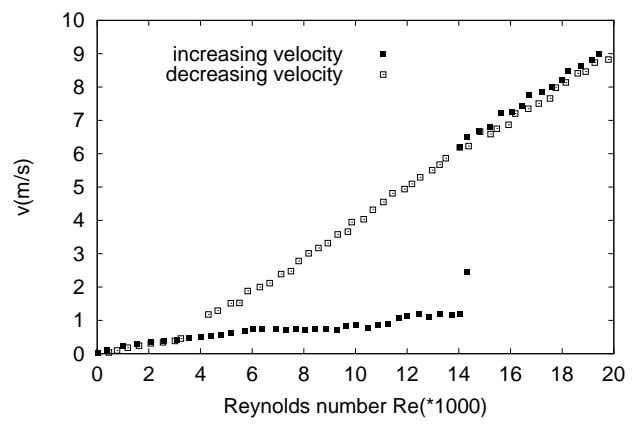

Figure 2. Evolution of the vertical velocity $V$ at $S_{2}$ with the Reynolds number Re at $\alpha=39^{\circ}$

cated in the duct at $S_{1}=-500 \mathrm{~mm}$ upstream from the exit, measures the mean velocity $U_{0}$ in the duct; the second one, situated in the jet axis at $S_{2}=100 \mathrm{~mm}$ downstream from the exit is used as a bifurcation indicator. Indeed, the hot-wire measures a velocity higher when the jet is aligned with the duct axis than when it is attached to the inclined plane. The Reynolds number is based on the nozzle height $h_{N}$ and velocity $U_{0}\left(R e=U_{0} h_{N} / v\right.$ where $v$ is the kinematic viscosity).

\section{Hysteresis loop}

The figure 2 shows that the vertical velocity $V$ measured by the downstream hot wire is almost null when the Reynolds number $R e$ increases from zero up to $R e_{c a} \simeq 14200$. But, at $R e_{c a}$, the $V$ value increases suddenly showing that the jet is now attached to the wall. When the jet is reattached, the velocity $V$ varies linearly with $R e$. When the Reynolds number decreases down to $R e_{c d} \simeq 3000\left(R e_{c d}<R e_{c a}\right)$, the vertical velocity suddenly drops indicating that the jet is detached. This experiment shows the hysteresis loop.

For $R e_{c d} \leq R e \leq R e_{c a}$ when the jet is attached (res. detached) to the wall, only a very large perturbation is able to detach (res. reattach) the jet. This indicates that both branches are stable. On the other hand whatever the perturbation is, the jet remains attached (res. detached) for $R e>R e_{c a}$ (res. $R e<R e_{c d}$ ). For this range of Reynolds numbers, there is one and only one stable branch. Moreover the bifurcation theory imposes an inflexion point at the Reynolds numbers $R e_{c a}$ and $R e_{c d}$. Consequently, it exists an unstable branch for $R e_{c d} \leq R e \leq R e_{c a}$. Thus the flow along an inclined wall presents a hysteresis with a stationary under critical bifurcation (see figure 3 ).

\section{Evolution of the bifurcation limits}

Two experiments were carried out in order to determine the bifurcation limits against $R e$ and $\alpha$. In the first set of experiments, we fixed $\alpha$ and we sought the attachment and detachment

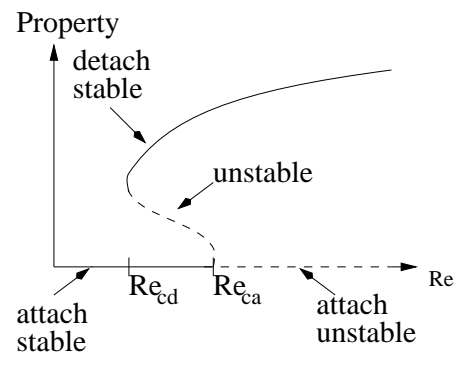

Figure 3. Bifurcation diagram

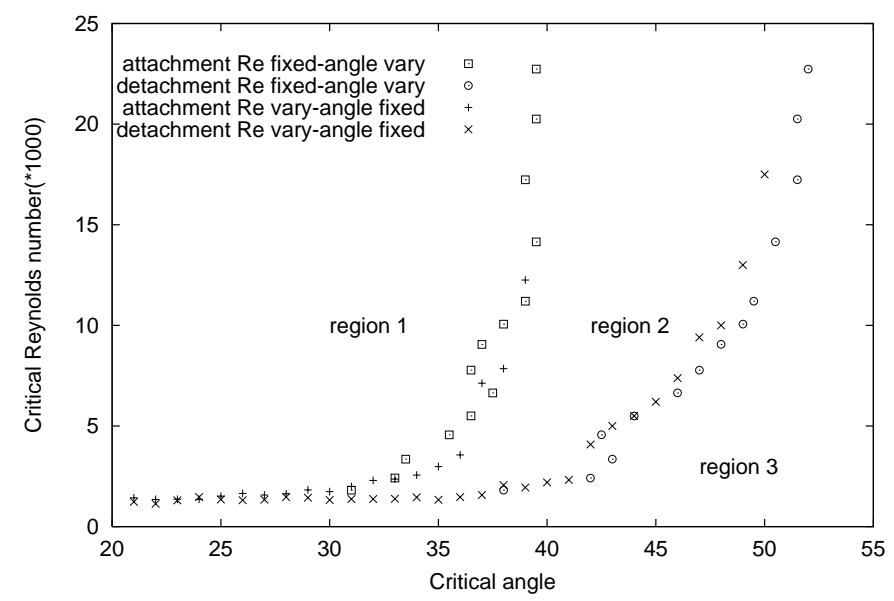

Figure 4. Flow regime for a jet in interaction with an inclined wall

critical Reynolds numbers $R e_{c a}$ and $R e_{c d}$. In the second set, we fixed the velocity and we measured the attachment and detachment critical angles $\alpha_{c a}$ and $\alpha_{c d}$. The figure 4 shows that both the experiments give the same limits of flow regime: in region 1 , the jet cannot be reattached to the wall; in region 3, the jet is always detached from the wall; in the intermediate zone 2 , the jet is reattached or detached depending on the variation of the parameters. Thus, for a given geometry $\left(L / h_{N}\right.$ fixed) there is a single couple of attachment parameters $\left(\alpha_{c a}, R e_{c a}\right)$ and a single couple of detachment parameters $\left(\alpha_{c d}, R e_{c d}\right)$.

For small angles $21^{\circ} \leq \alpha \leq 32^{\circ}$ the Reynolds number of attachment $R e_{c a}$ increases slightly (1350 to 3000). From $\alpha=$ $33^{\circ}, R e_{c a}$ increases rapidly and beyond $\alpha=38^{\circ}$ the jet attaches to the wall for large Reynolds numbers $(>10000)$. In the same way, for $21^{\circ} \leq \alpha \leq 38^{\circ}$, the Reynolds number of detachment $R e_{c d}$ increases slightly (1200 to 2100) and strongly for $\alpha>38^{\circ}$.

Furthermore the hysteresis zone characterised by $\Delta R e=R e_{c a}-R e_{c d}$ increases with the angle $\alpha$ (150 for $\alpha=21^{\circ}$ to 8000 for $\alpha=38^{\circ}$ ). When velocity is constant, the 


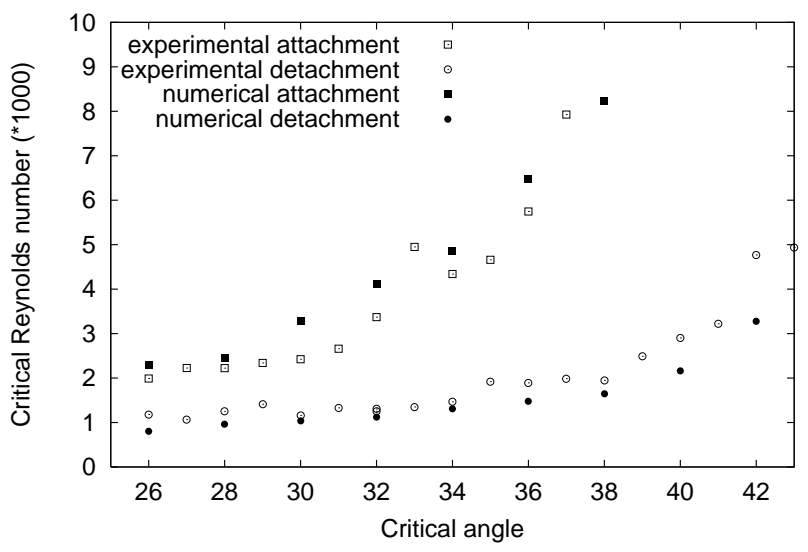

Figure 5. Comparison between the critical Reynolds numbers obtained experimentally and by simulation

hysteresis zone described by $\Delta \alpha=\alpha_{c a}-\alpha_{c d}$ increases with the $\operatorname{Re}\left(11^{\circ}\right.$ for $\operatorname{Re}=10000$ and $7^{\circ}$ for $\left.\operatorname{Re}=1800\right)$.

Our attachment and detachment curves are in agreement with Newman's results (Newman, 1961). For $R e>10000$ the attachment and detachment angles vary indeed very little. Moreover, our attachment angle has the same order of magnitude as those measured by Newman. Nevertheless, the detachment angle is different: $\alpha_{c d} \simeq 58^{\circ}$ for Newman and $\alpha_{c d} \simeq 50^{\circ}$ in our case. This difference could be explained by the nozzle shape at the exit, not rounded in our experiment.

\section{Divergent configuration}

Similar experiments were carried out on a divergent configuration (i.e. a second wall is positioned symmetrically to the first one). This configuration shows a Coanda effect with a hysteresis loop. The angles and Reynolds numbers of attachment $\left(\alpha_{c a}, R e_{c a}\right)$ and detachment $\left(\alpha_{c d}, R e_{c d}\right)$ are similar to these showed previously with a single inclined wall. For a symmetric divergent, the jet reattaches randomly to one or the other wall. Thus, the bifurcation diagram shown in figure 3 has to be completed by symmetry with the Reynolds-axis.

\section{NUMERICAL SIMULATION OF THE DIVERGENT}

Large eddy simulations (LES) (Sagaut, 1998), based on the Smagorinsky's model with an additional equation for the turbulent kinetic energy were achieved on the divergent configuration. In order to avoid a numerical return which would perturb the flow in the divergent, a large cavity was added at the divergent exit as shown in figure 6. The structured mesh was composed of 10000 cells. The flow was advanced in time by using a blended Cranck-
Nicholson method with a numerical time step $\Delta t=0.001 \mathrm{~s}$ on a interval of time of 20s. The spatial discretisation used a MARS scheme and the problem was solved with a PISO algorithm. At the inlet, a boundary velocity with a turbulent intensity of $10 \%$ was imposed. On the divergent wall, a no-slip condition was applied and we imposed a pressure condition on the others boundaries.

We performed simulations on a time interval of 20 s for various angles of inclination. We noted the following behaviour. Below a Reynolds number close to the experimental value of the detachment Reynolds number $R e_{c d}$, the jet is always detached from the wall during the simulation. On the contrary, above a Reynolds number close to experimental value of the reattachment Reynolds number $R e_{c a}$, the jet is always reattached to the wall during the simulation. And for $R e_{c d}<R e<R e_{c a}$, the jet is initially detached but becomes attached to the wall under the impulse of the numerical perturbations. This last result agrees with experiments showing that for $R e_{c d}<R e<R e_{c a}$, a perturbation leads to the jet attachment. So, we defined numerical Reynolds numbers of detachment as being the Reynolds of the first test where the jet is no more axial at the end of the simulation and the number of Reynolds of attachment as being the Reynolds of the first test where the jet is attached to a wall of divergent from the beginning of the simulation.

As shown in figure 5, numerical and experimental curves have a similar shape. The quantitative difference can be explained by a slightly dissymmetry of the divergent in our experiments. Furthermore, here, the Reynolds numbers of bifurcation are not determined by following the branches of solution as in the case of the method of continuation. Actually, we do several simulations to find by dichotomy the critical Reynolds. So the results obtained give an interval, but do not give the critical Reynolds with precision.

\section{CONSTRUCTION OF A DYNAMICAL SYSTEM BY POD METHOD \\ Proper orthogonal decomposition}

The Proper orthogonal decomposition (POD), which was first introduced to the fluid mechanics society by Lumley (1967), is a powerful technique for extracting basis functions that represent ensemble averaged structures. Here, we give a short description of the POD method; for more details, see (Sirovich, 1987).

The POD method allows to construct a complete basis of $L^{2}(\Omega)$. This basis is obtained to solve the Fredholm integral equation: ${ }^{1}$

$$
\int_{\Omega} R\left(x, x^{\prime}\right) \phi\left(x^{\prime}\right) d x^{\prime}=\lambda \phi(x)
$$

\footnotetext{
${ }^{1}$ In practice for data issued of simulations the construction of the basis $\phi$ uses the Snapshot method (Sirovich, 1987)
} 


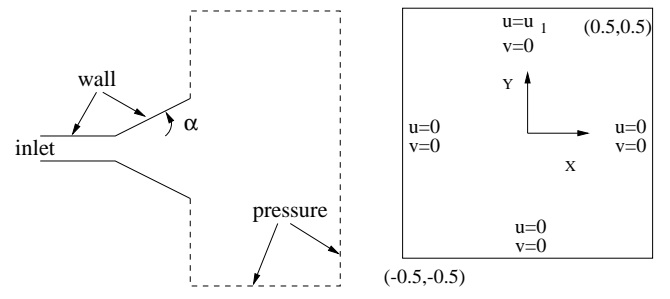

Figure 6. Divergent scheme and cavity scheme

representing an eigenvalue problem for $\phi . R$ is the fluctuating spatial correlation tensor defined by:

$$
R_{i j}\left(x, x^{\prime}\right)=\overline{u_{i}^{\prime}(x) u_{j}^{\prime}\left(x^{\prime}\right)}
$$

As $R$ is symmetric and positive, the eigenfunctions $\phi_{n}$ are orthogonal according to the Hilbert Schmidt's theory. Each eigenvalue $\lambda_{n}$, taken individually, represents the energy contribution of the corresponding eigenfunction. The sum of these eigenvalues, numbered such that $\lambda_{n} \geq \lambda_{n+1}$ represents the energy of the stochastic signal. The energy-optimal transformation property of POD is exploited when truncating the set of POD modes by keeping the first $m$ modes and ignoring the remaining modes. We obtain the reduced-order model of the form

$$
u^{\prime}(x, t)=\sum_{n=1}^{m} a_{n}(t) \phi_{n}(x)
$$

\section{Galerkin projection-Dynamical system}

The first model obtained by the POD basis capable of reproducing realistic low dimensional dynamics of a turbulent flow system was presented in the pioneering paper of Aubry et al. (1988). Since, this method has been applied for a turbulent driven-cavity by Cazemier et al. (1998), for turbulent channel flow by Omurtag et al. (1999)...

We decompose the velocity $u$ (res. the pressure $p$ ) into the mean velocity $\bar{u}$ (res. the mean pressure $\bar{p}$ ) and the fluctuating velocity $u^{\prime}$ (res. the fluctuating pressure $p^{\prime}$ ). Using the POD decomposition (3), we obtain

$$
u(x, t)=\bar{u}+\sum_{n=1}^{m} a_{n}(t) \phi_{n}(x)
$$

The Galerkin projection of the fluctuating Navier-Stokes equations on the spatial structures $\phi_{n}$, taking $\phi$ orthonormal and the divergence of $\phi$ null, is written:

$$
\frac{d a_{n}}{d t}=D_{n}+E_{n}+F_{n}+\sum_{m=1}^{N_{g a l}} B_{n m} a_{m}+\sum_{m=1}^{N_{g a l}} \sum_{k=1}^{N_{g a l}} C_{n m k} a_{m} a_{k}
$$

Table 1. SOME CHARACTERISTIC EIGENVALUES OF THE POD

\begin{tabular}{ccc}
\hline mode $i$ & $\lambda_{i}$ & \% fluctuating energy \\
\hline 1 & $0.11832 \mathrm{E}-03$ & 45.647 \\
2 & $0.11219 \mathrm{E}-03$ & 88.931 \\
5 & $0.49213 \mathrm{E}-05$ & 95.862 \\
10 & $0.51169 \mathrm{E}-06$ & 99.534 \\
\hline
\end{tabular}

with $n=1, \ldots, N_{\text {gal }}$ where $N_{\text {gal }}$ is the Galerkin mode number. The coefficients of (5) are defined by :

$$
\begin{aligned}
& B_{n m}=\left(\phi_{n}, \frac{1}{\operatorname{Re}} \Delta \phi_{m}-\operatorname{Grad} \bar{u} \cdot \phi_{m}-\operatorname{Grad} \phi_{m} \cdot \bar{u}\right) \\
& F_{n}=\left(\phi_{n},-G r a d \bar{u} \cdot \bar{u}\right) \quad D_{n}=-\int_{\partial \Omega} p^{\prime} \phi_{n} . n_{o} d S \\
& E_{n}=\left(\phi_{n},-\operatorname{grad} \bar{p}+\frac{1}{R e} \Delta \bar{u}\right) \quad C_{n m k}=-\left(\phi_{n}, \operatorname{Grad} \phi_{m} . \phi_{k}\right)
\end{aligned}
$$

In the dynamical system, it is very difficult to know the pressure ${ }^{2}$ giving $D_{n}$. However, in many situations this term is null. Otherwise, we have to model this term.

The coefficients $B_{n m}, C_{n m k}, D_{n}$ and $E_{n}$ are discretised on the LES grid and the spatial derivatives are discretised by finite difference. The integrations over the flow $\Omega$ are approximated using the trapezoïdal rule for numerical integration. Temporal integration of discrete dynamical System is solved by the classical fourth-order Runge-Kutta method.

\section{Validation to a lid driven cavity}

We validated our algorithm on two configurations: on a laminar unsteady channel flow and on a turbulent lid driven cavity flow. In this paper we present the POD method and the low dimensional model construct of the flow in a lid driven cavity. In the latter case, the fluctuating velocity is null on the boundaries, so $D_{n}$ vanish.

The flow field data for POD method of driven cavity are computed by LES as described above. The grid size is $100 * 100$. At the top of the square cavity of size $a_{1}=1 \mathrm{~m}$, we impose a parabolic profile with a maximal velocity $U_{0}$ giving a $\operatorname{Re}=$ $100000\left(\operatorname{Re}=U_{0} a_{1} / \mathrm{v}\right)$. On the other boundaries, the velocities are null (see figure 6).

To construct the POD basis, we used 90 snapshots separated of a $0.1 \mathrm{~s}$ step time. This number of snapshots was chosen as large as possible for an accurate computation of the POD. As shown in the table 1 , the first eigenfunctions contain most of the

\footnotetext{
${ }^{2}$ It is not possible to decompose : $p^{\prime}=\sum_{n=1}^{N_{g a l}} a_{n}(t) p_{n}(x)$
} 


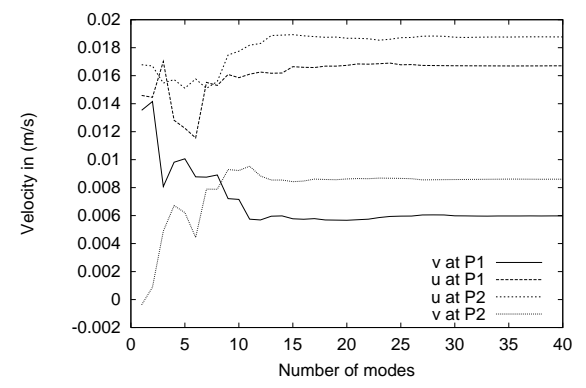

Figure 7. Evolution of vertical and horizontal fluctuating velocities at $P 1=(-0.2,-0.175)$ and at $P 2=(0.175,0.3)$ against the number of $P O D$ 's modes (for $t=0.55 \mathrm{~s}$ )

fluctuating kinetic energy. Ten modes are sufficient to capture 99\% of the energy. More over, the effect of truncation can be also viewed in figure 7 . The evolution of the reconstruction of the fluctuating velocities given by (3) against the number of POD modes in two points at $t=0.55 \mathrm{~s}$ is plotted. From 15 modes, the reconstruction of the fluctuating velocities is almost constant. This remark and the energetic consideration indicate that with only 15 modes, the velocity field is rather well reconstructed. So, the first 15 modes were used for constructing a low-dimensional model according to (5). In figure 8, we present the temporal evolution of the temporal projection obtained by the dynamical system (DS) and we compare them with those obtained by POD. We note that DS modes evolve in the same way as the modes of POD.

\section{CONCLUSION AND FUTURE DIRECTIONS}

The flow along an inclined wall can be axial or deflected toward the wall, depending on the values of the Reynolds number $R e$ and the inclination angle $\alpha$. In this paper we note that:

1. a hysteresis loop is associated to this phenomenon

2. there is one and only one couple of parameters of attachment $\left(\alpha_{c a}, R e_{c a}\right)$ and one and only one couples of parameters of detachment $\left(\alpha_{c d}, R e_{c d}\right)$.

3. as $\alpha$ increases, the Reynolds of attachment $R e_{c a}$ and the Reynolds of detachment $R e_{c d}$ increases.

Experimental results indicate also that the behaviour is the same when a second wall is symmetrically added (divergent configuration). The LES simulations performed on this configuration are qualitatively in agreement with the experimental data but there is a quantitative difference.

To apprehend the Coanda effect, we want to construct a lowdimensional dynamical system by using a Proper Orthogonal Decomposition (POD). In this paper, we valid in a square cavity the construction of the dynamical system. This dynamical sys-

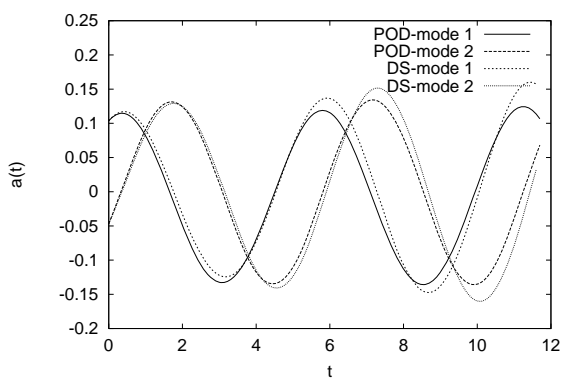

Figure 8. Comparison between POD and dynamical system modes

tem will use to study the bifurcation diagram for the divergent configuration. Forward, our objective is an optimal control of parameters to obtain the maximum of noise reduction.

\section{REFERENCES}

Aubry, N., Holmes, P., Lumley, J.L. and Stone, E. The $d y$ namics of coherent structures in the wall region of a turbulent boundary layer, J. Fluid Mech., 192, 115-173, 1988.

Bradshow, P. and Wong, F.Y.F. The reattachment and relaxation of turbulent shear layer, J. Fluid Mech., 52, 113-135, 1972.

Cazemier, W., Verstappen, R.W., and Veldman, A.E. Proper orthogonal decomposition and low-dimensional models for driven cavity flow, Phys. Fluids, 10(7), 1685-1699, 1998.

Katz, P., Der Coanda-Effekt, Gesundheits-Ingenieur, 6, 169192, 1973.

Lumley, J.L. The structure of inhomogenous turbulent flows, Atmospheric Turbulence and Radio Wave Propagation, Yaglom A.M. and Tatarski V.I, 167-178, 1967.

Guérin S., Reduction de sons auto-entretenus par effet Coanda sur des jets auxiliaires, Thesis, La Rochelle, 2001.

Guérin, S., Allery, C. and Sakout, A. Hystérésis et réduction de sons auto-entretenus commandées par effet Coanda, C. R. Acad. Sci. série IIb, 329(8), 579-584, 2001.

Lai, J.C.S. and Lu, D. Effect of wall inclination on the mean flow and turbulence characteristics in a two-dimensional wall jet, Int. J. Heat and Fluid Flow, 17, 377-385, 1996.

Newman, B.G., The deflexion of plane jets by adjacent boundaries - Coanda effect, Boundary Layer and Flow control, G. V. Lachmann (ed.), Pegamon Press New York, 232-265, 1961.

Omurtag, A. and Sirovich, L. On low-dimensional Modeling of Channel turbulence, Theo. Comp. Fluid Dyn., 13, 115-127, 1999.

Sagaut, P., Introduction à la simulation des grandes échelles, Springer, 1998.

Sirovich, L., Turbulence and the dynamics of coherent structures Part 1, Quat. App. Math., 16(3), 561-571, 1987. 